\title{
Diagnostic Biomarkers in Cerebrospinal Fluid in Primary Central Nervous System Lymphoma: A Protocol for Systematic Review and Meta-Analysis
}

\section{Lili Zhou}

General Hospital of Western theater Command

Hai Yi ( $\square$ yihaimail@163.com )

General Hospital of Western Theater Command

\section{Dan Chen}

General Hospital of Western Theater Command

Qian Zhang

General Hospital of Western Theater Command

\section{Fangyi Fan}

General Hospital of Western Theater Command

Ling Qiu

General Hospital of Western Theater Command

Nan Zhang

General Hospital of Western Theater Command

Yi Su

General Hospital of Western Theater Command

\section{Protocol}

Keywords: Diagnosistic biomarkers, Cerebrospinal fluid, Primary central nervous system lymphoma, Systematic review, Meta-analysis

Posted Date: December 17th, 2020

DOI: https://doi.org/10.21203/rs.3.rs-128291/v1

License: (c) (i) This work is licensed under a Creative Commons Attribution 4.0 International License. Read Full License 


\section{Abstract}

\section{Background}

Primary central nervous system lymphoma (PCNSL) is a highly aggressive non-hodgkin's lymphoma with unfavorable prognosis. Currently, the diagnosis of PCNSL relies on brain excisional biopsy, which is an invasive procedure, carries the risk of complications such as intracranial hemorrhage and functional impairment. Finding effective biomarkers will help us to diagnose PCNSL faster and safer.

\section{Methods}

This systematic review will be conducted in accordance with the Preferred Reporting Items for Systematic Reviews and Meta-Analyses Protocol (PRISMA-P) 2015 guideline.We will search databases including PubMed, Cochrane Library, Medline, Web of Science, EMBASE and CNKI. The studies that demonstrated the diagnostic value of certain biomarkers in CSF for PCNSL will be included. For quantitative value, the standardized mean diffenence (SMD) and their $95 \%$ confidence intervals (CI) will be calculated. The outcomes are the mean difference of biomarker levels in CSF between PCNSL patients and controls.

\section{Discussion}

In this systematic review we will analyze the studies of the biomarkers in cerebrospinal fluid for diagnosis of PCNSL. This research can help us to identify the biomarkers with diagnostic value for PCNSL, making diagnosis of PCNSL easier, faster and safer.

\section{Systematic review registration}

PROSPERO CRD42020218143.

\section{Background}

Primary central nervous system lymphoma (PCNSL) is a raresubtype of non-Hodgkin's lymphoma, tend to occur in older people with a median age of 65 years old[1]. The common primary sites are the brain, leptomeninges, spinal cord, and eyes, without infiltration of other parts. PCNSL can occur in immunocompromised individuals such as HIV-infected hosts, post organ transplant recipient[2]. In recent years, more and more cases in immunocompetent hostshave been reported[3]. PCNSL is particularly theactivated $B$ cell-like $(A B C)$ subtype, constitutive activation of the nuclear factor kappa-light-chainenhancer of activated B cells (NF-KB) pathway, mostly accompanied by myeloid differentiation factor 88 (MyD88) and CD79B mutations[4]. PCNSL is a highly aggressive malignant lymphoma with unfavorable prognosis[5]. It responds poorly to conventional chemotherapy, and the overall 5-year survival rate is only about 33\%[5]. Combination chemotherapy with high-dose methotrexate, whole brain radiotherapy, and autologous hematopoietic stem cell transplantation can benefit PCNSL patients[6-9]. In recent years, novel targeted therapeutics have been emerging as potential treatments for PCNSL[10]. For instance, bruton tyrosine kinase (BTK) inhibitor ibrutinib significantly improves the prognosis of PCNSL, especially 
for patients with MyD88 and CD79B double mutations[11]. The immunomodulator lenalidomide also enhance the efficacy of current treatment in PCNSL[12].

The clinical manifestations of PCNSL patients are nausea, headache, vomiting, limb weakness, et al[1]. Imaging examinations such as computed tomography (CT) and Magnetic resonance imaging (MRI) can find intracranial space-occupying lesions. Several studies have reported that PCNSL has certain changes in imaging features, such as the lesions are mostly located in the supratentorial area, with MRI hypointense on T1-weighted images, iso-hypointense on T2-weighted images, homogeneous enhancement and restricted diffusion, little associated vasogenic edema, no central necrosis, which can be distinguished from other tumor types such as glioma[13]. However, brain excisional biopsy is still the gold standard for diagnosis. Nevertheless, brain biopsy is an invasive procedure carries the risk of complications such as intracranial hemorrhage and functional impairment[14, 15]. The procedure may also be challenging because of the difficulty in reaching the deep tumor sites[16]. Exploring highefficiency and less invasive diagnostic strategy is an urgent need for the diagnosis of PCNSL.

Recently, several studies show that certain diagnostic biomarkers could be detected in cerebrospinal fluid (CSF) and peripheral blood in PCNSL patients $[17,18]$, providing ideas for the diagnosis of PCNSL without brain excisional biopsy. These biomarkers include tumor cells, tumor DNAs and proteins. The CSF biomarkers have better sensitivity and auxiliary diagnostic value on PCNSL than the blood biomarkers[19]. Because lumbar puncture is routine and less traumatic in patients with intracranial lesions, CSF biomarkers are potentially the most suitable biomarkers for PCNSL diagnosis.

\section{Objectives}

The aim of this systematic review and meta-analysis is to evaluate the diagnostic performance of the frequently reported biomarkers in CSF for PCNSL patients.

\section{Methods}

This systematic review was registered on the International Prospective Register of Systematic Reviews (PROSPERO) (CRD42020218143) on the $29^{\text {th }}$ of November 2020. This systematic review will be conducted in accordance with the Preferred Reporting Items for Systematic Reviews and Meta-Analyses Protocol (PRISMA-P) 2015 guideline. (Additional file 1).

\section{Eligibility criteria}

\section{Type of studies}

This review will include all types of studies with human subjects that evaluate the diagnostic value of biomarkers in CSF in PCNSL. Case reports, letters, animal studies and laboratory studies will be excluded.

\section{Type of participants}


Patients with PCNSL diagnosis according to histopathology will be included, regardless of gender, age, race, ethnicity, involvement site, host immune status and severity of PCNSL. The patients with secondary central nervous system lymphoma would be excluded.

\section{Type of index test}

Index test: CSF-based tumor cells, tumor DNA and proteins were used in the detection of PCNSL. Whereas case reports, reviews, in vitro studies will be excluded.

\section{Types of intervention}

Several cytokines and genemutations in CSF were reported to associated with diagnosis of PCNSL. The concentration or properties of the following biomarkers in CSF of PCNSL patients and controls will be evaluated in this study.

Biomarkers: MiR-21, miR-19, miR-30c, miR-92a, transmembrane activator and calcium modulator and cyclophilin ligand interactor (TACI), B-cell maturation antigen (BCMA), soluble IL-2 receptor (SIL-2R), interleukin-6 (IL-6), interleukin-10 (IL-10), $\beta 2$-microglobulin ( $\beta 2-M G)$, the C-X-C motif chemokine ligand 13 (CXCL13), T-cell immunoglobulin and mucin domain 1 (Tim-1), neopterin (Npt), a proliferation inducing ligand (APRIL), B cell activating factor (BAFF), CD79B, myeloid differentiation factor 88 (MYD88), circulating U2 small nuclear RNA fragments (RNU2-1f), soluble CD27, osteopontin (OPN), Antithrombin III (AT III).

\section{Types of outcome measures}

Diagnostic value of certain biomarkers in CSF in the diagnosis of PCNSL.

Mean differenceof biomarker levels in CSF between PCNSL patients and controls.

\section{Data sources and search strategy}

A literature search will be performed in multiple electronic databases including PubMed, Cochrane Library, Medline, Web of Science, EMBASE and CNKI from their inception to October 30, 2020. There are no language restrictions. The search strategy of Medline was shown in Additional file 2. Other databases will be used by similar strategy.

\section{Data collection and analysis}

\section{Study selection}

Two reviewers (FF and LQ) will evaluate the titles and abstracts by searching information sources independently. The fulltext of potential article will be assessed by two reviewers if the eligibility of an article could not be decided by only screening the title and abstract. Any disagreements regarding eligibility of studies will be adjudicated by discussion with a third reviewer (YS). 


\section{Data extraction}

Two reviewers ( $\mathrm{HY}$ and FF) will extract the data from each included study independently by using a standardized form. The items extracted from the studies are first author, study title, publication year, regions, study design, sample size and controls, detection methods, biomarker and levels, diagnostic value, value type, sensitivity and specificity. If there are missing data, we will contact the authors for additional information. Any disagreements will be resolved through discussion and the standardized forms will be checked by NZ.

\section{Risk of bias in individual studies}

The quality of included studies and risk of bias will be assessed independently by two reviewers (HY and FF) using Quality Assessment of Diagnostic Accuracy Studies (QUADAS-2) criteria[20]. The quality assessment evaluate the risk of bias and concerns regarding applicability of all included studies. The results of quality assessment and studies with a high, low, or unclear risk of bias will be displayed in a table.

\section{Data synthesis}

\section{Statistical analysis}

The mean and standard deviation of biomarker levels from individual studies will be collected to calculate the standardized mean difference (SMD) and their 95\% confidence interval (Cl). Heterogeneity will be evaluated by the Cochran's $Q$ Test and the inconsistency index value $\left(/^{2}\right)$. If there is no heterogeneity $\left(R^{2}<40 \%, \mathrm{p}>0.05\right)$, we will use fixed-effects model for meta-analysis; otherwise, the randomeffects model would be chosen[21]. All the statistical analysis will be conducted using the STATA version 15.0.

\section{Subgroup analysis}

To further understand the heterogeneity, we will perform subgroup analyses as follows,

1. The type of biomarkers (tumor cells, tumor DNA and proteins).

2. Detection methods (Droplet Digital PCR, Next Generation Sequencing, ELISA).

\section{Sensitivity analysis}

To determine the stability of the study, we will perfrom sensitivity analysis. The studies with high risk of bias on overall effects will be excluded.

\section{Assessment of reporting bias}

Funnel plots, as well as associated regression tests, will be used to test publication bias. 


\section{Confidence in cumulative evidence}

The Grading of Recommendations, Assessment, Development, and Evaluation (GRADE) will be used to assess the strength of evidence.

\section{Discussion}

We will evaluate the role of all the reported biomarkers in CSF in patients with PCNSL in this systematic review and meta-analysis. Also we will clarify the optimal concentrations of certain biomarkers for the diagnosis. By subgroup analyses, we would compare the different type of biomarkers and the different detection methods for the diagnosis of PCNSL. We hope the findings of this study will help us to recognize the significance of liquid biopsy in CSF for PCNSL patients, making diagnosis of PCNSL easier, faster and safer, andgetting better quality of life.

\section{Abbreviations}

PCNSL: Primary central nervous system lymphoma

CSF: Cerebrospinal fluid

ABC: Activated B cell-like

NF-KB: Nuclear factor kappa-light-chain-enhancer of activated B cells

BTK: Bruton tyrosine kinase

CT: computed tomography

MRI: Magnetic resonance imaging

PRISMA-P: Preferred Reporting Items for Systematic Review and Meta-Analysis Protocols

QUADAS-2: Quality Assessment of Diagnostic Accuracy Studies

DNA: Deoxyribonucleic acid

PCR:Polymerase chain reaction

ELISA: Enzyme-linked immunosorbent assay

\section{Declarations}

\section{Ethics approval and consent to participate}


This study is a systematic review, the results are based on previously published evidence. So there is no need for ethics.

\section{Consent for publication}

Not applicable.

\section{Availability of data and materials}

Not applicable.

\section{Competing interests}

The authors declare that they have no competing interests.

\section{Funding}

This work is supported by the Science and Technology Project of Sichuan (No. 2018JY0583), Scientific Research Project of Sichuan Health Commission (No.18PJ358) and Youth Innovation Project of Sichuan Medical Research (No.Q17004). The sponsors are not involved in design, data collection, data analysis and interpretation, or writing the manuscript.

\section{Authors' contributions}

LZ, DC, and QZ helped conceive the idea. LZ and HY designed the study and drafted the protocol. FF, LQ and YS designed the search strategy. HY, FF, and NZ designed the data extraction and statistical analysis. HY wrote the manuscript. All authors approved the final manuscript.

\section{References}

1. Citterio G, Reni M, Gatta G, Ferreri AJM. Primary central nervous system lymphoma. Critical reviews in oncology/hematology. 2017;113:97-110.

2. Gupta NK, Nolan A, Omuro A, Reid EG, Wang CC, Mannis G, et al. Long-term survival in AIDS-related primary central nervous system lymphoma. Neuro-oncology. 2017;19(1):99-108.

3. Grommes C, DeAngelis LM. Primary CNS Lymphoma. Journal of clinical oncology : official journal of the American Society of Clinical Oncology. 2017;35(21):2410-8.

4. Ho KG, Grommes C. Molecular profiling of primary central nervous system lymphomas - predictive and prognostic value? Current opinion in neurology. 2019;32(6):886-94.

5. Han $\mathrm{CH}$, Batchelor TT. Diagnosis and management of primary central nervous system lymphoma. Cancer. 2017;123(22):4314-24.

6. Kasenda B, Schorb E, Fritsch K, Finke J, Illerhaus G. Prognosis after high-dose chemotherapy followed by autologous stem-cell transplantation as first-line treatment in primary CNS lymphoma--a 
long-term follow-up study. Annals of oncology : official journal of the European Society for Medical Oncology / ESMO. 2012;23(10):2670-5.

7. Martinez-Calle N, Poynton E, Alchawaf A, Kassam S, Horan M, Rafferty M, et al. Outcomes of older patients with primary central nervous system lymphoma treated in routine clinical practice in the UK: methotrexate dose intensity correlates with response and survival. British journal of haematology. 2020;190(3):394-404.

8. Holdhoff M, Ambady P, Abdelaziz A, Sarai G, Bonekamp D, Blakeley J, et al. High-dose methotrexate with or without rituximab in newly diagnosed primary CNS lymphoma. Neurology. 2014;83(3):235-9.

9. Houillier C, Taillandier L, Dureau S, Lamy T, Laadhari M, Chinot O, et al. Radiotherapy or Autologous Stem-Cell Transplantation for Primary CNS Lymphoma in Patients 60 Years of Age and Younger: Results of the Intergroup ANOCEF-GOELAMS Randomized Phase II PRECIS Study. Journal of clinical oncology : official journal of the American Society of Clinical Oncology. 2019;37(10):823-33.

10. Illerhaus G, Schorb E, Kasenda B. Novel agents for primary central nervous system lymphoma: evidence and perspectives. Blood. 2018;132(7):681-8.

11. Grommes C, Younes A. Ibrutinib in PCNSL: The Curious Cases of Clinical Responses and Aspergillosis. Cancer cell. 2017;31(6):731-3.

12. Ghesquieres $\mathrm{H}$, Chevrier $\mathrm{M}$, Laadhari $\mathrm{M}$, Chinot $\mathrm{O}$, Choquet $\mathrm{S}$, Molucon-Chabrot $\mathrm{C}$, et al. Lenalidomide in combination with intravenous rituximab (REVRI) in relapsed/refractory primary CNS lymphoma or primary intraocular lymphoma: a multicenter prospective 'proof of concept' phase II study of the French Oculo-Cerebral lymphoma (LOC) Network and the Lymphoma Study Association (LYSA)dagger. Annals of oncology : official journal of the European Society for Medical Oncology / ESMO. 2019;30(4):621-8.

13. Cheng G, Zhang J. Imaging features (CT, MRI, MRS, and PET/CT) of primary central nervous system lymphoma in immunocompetent patients. Neurological sciences : official journal of the Italian Neurological Society and of the Italian Society of Clinical Neurophysiology. 2019;40(3):535-42.

14. van der Meulen M, Dirven L, Habets EJJ, van den Bent MJ, Taphoorn MJB, Bromberg JEC. Cognitive functioning and health-related quality of life in patients with newly diagnosed primary CNS lymphoma: a systematic review. The Lancet Oncology. 2018;19(8):e407-e18.

15. Khatab S, Spliet W, Woerdeman PA. Frameless image-guided stereotactic brain biopsies: emphasis on diagnostic yield. Acta neurochirurgica. 2014;156(8):1441-50.

16. Kerbauy MN, Moraes FY, Lok BH, Ma J, Kerbauy LN, Spratt DE, et al. Challenges and opportunities in primary CNS lymphoma: A systematic review. Radiotherapy and oncology : journal of the European Society for Therapeutic Radiology and Oncology. 2017;122(3):352-61.

17. Yu X, Li Z, Shen J, Chan MT, Wu WK. Role of microRNAs in primary central nervous system lymphomas. Cell proliferation. 2016;49(2):147-53.

18. Hiemcke-Jiwa LS, Minnema MC, Radersma-van Loon JH, Jiwa NM, de Boer M, Leguit RJ, et al. The use of droplet digital PCR in liquid biopsies: A highly sensitive technique for MYD88 p.(L265P) detection in cerebrospinal fluid. Hematological oncology. 2018;36(2):429-35. 
19. Bobillo S, Crespo M, Escudero L, Mayor R, Raheja P, Carpio C, et al. Cell free circulating tumor DNA in cerebrospinal fluid detects and monitors central nervous system involvement of B-cell lymphomas. Haematologica. 2020.

20. Whiting PF, Rutjes AW, Westwood ME, Mallett S, Deeks JJ, Reitsma JB, et al. QUADAS-2: a revised tool for the quality assessment of diagnostic accuracy studies. Annals of internal medicine. 2011;155(8):529-36.

21. Kumarasamy C, Devi A, Jayaraj R. Prognostic value of microRNAs in head and neck cancers: a systematic review and meta-analysis protocol. Systematic reviews. 2018;7(1):150.

\section{Supplementary Files}

This is a list of supplementary files associated with this preprint. Click to download.

- AdditionalFile1PRISMAP2015Checklist.docx

- AdditionalFile1PRISMAP2015Checklist.docx

- AdditionalFile2Searchstrategy.docx

- AdditionalFile2Searchstrategy.docx 